\title{
Literaturadaptionen in der Graphic Novel Eine weitere Axt für das gefrorene Meer in uns?
}

\author{
Mag. . Caroline Bader, Universität Innsbruck
}

\begin{abstract}
Als Franz Kafka 1904 an Oskar Pollak schrieb, ein Buch müsse wie „die Axt sein für das gefrorene Meer in uns" ${ }^{\text {1 }}$, bezog er sich dabei auf jene Werke, denen er ein hohes Potenzial zuschrieb, etwas tief im Inneren der Menschen zu berühren. Literatur, die eine solch brachiale Wirkung auf uns ausübt, bricht etwas auf, dringt (pathetisch gesprochen) zu etwas in unserem Inneren vor, hält uns zu intensiver Lektüre an und bewirkt nachhaltiges literarisches Lesen; nur diese Werke waren es Kafka wert, gelesen zu werden.

Als Klassiker der ,Weltliteratur' genießen Kafkas Erzählungen kanonischen Status an Österreichs Schulen. Dass jedes seiner Werke $\mathrm{zu}$ intensivem, nachhaltigem literarischen Lernen anhalten kann und so gewissermaßen eine ,Axt' darstellt, die das Meer in uns aufzubrechen vermag, dürfte insofern heute kaum noch jemand bezweifeln. Für Literaturcomics und -Graphic Novels gilt dies in dieser Form noch nicht. Diese Formate leiden nach wie vor unter dem Makel des Trivialliterarischen, wodurch es in den häufigsten Fällen nicht zu einer schüler- und lehrerseitigen Auseinandersetzung mit dem Inhalt kommt, weil der Einbezug in den Unterricht schon am ,Trägermedium“ scheitert. Darüber hinaus fühlen sich eine große Anzahl von Lehrenden mit dem
\end{abstract}

1 Brief an Oskar Pollak vom 27. Jänner 1904, in: Briefe 1902-1924, Hrsg. von Max Brod. Frankfurt a.M.: 1966.

Kapelari, Suzanne (Hg.), Vierte „Tagung der Fachdidaktik“ 2019:

„Interdisziplinäre fachdidaktische Diskurse zur Bildung für nachhaltige Entwicklung“

(C) 2020 innsbruck university press, ISBN 978-3-99106-019-2, DOI 10.15203/99106-019-2 
Medienformat überfordert und zweifeln an ihrer eigenen Kompetenz hinsichtlich einer Auseinandersetzung mit diesen Transformationen, was womöglich ebenfalls eine Hürde darstellt, ihnen im Unterricht zu begegnen. Solchen Annahmen soll dieser Beitrag entgegenwirken, indem er das Potenzial der Transformation von Franz Kafkas Die Verwandlung auslotet, um damit ein Urteil über die Axt-Kompetenz zumindest einer Adaption fällen zu können.

\section{Comics und Graphic Novels}

Im Grunde handelt es sich bei Comics und Graphic Novels um Formate moderner Bildgeschichten, die weder einem narrativen Einzelbild noch einem illustrierten Verbaltext entsprechen. Durch den Einsatz unbewegter, sich aufeinander beziehender Einzelbilder in Form eines Printmediums grenzt sich dieses Format ferner von Zeichentrickfilmen oder den neuesten digitalen Medien ab. Der Comic als Terminus schließt dabei in den meisten Fällen die spezifischen Spielarten Graphic Novel und Manga mit ein und besteht (zumeist) aus einem verbalen und piktoralen Zeichensystem, in deren funktionalem Zusammenspiel eine zentrale Eigenart dieses Formats $\mathrm{zu}$ finden ist (DolleWeinkauff, 2014). Obgleich diese Text-Bild-Kombinationen ein essenzielles Mittel der Narration darstellen, sind sie keineswegs ein obligatorisches; Charakteristisch für diese Formate ist die fakultative Möglichkeit dieser Interdependenzen (Palmier, 2015). Der sequentielle, an die Leserichtung der deutschen Schriftsprache orientierte Aufbau ist dabei gleichsam konstituierend wie die relativ unselbstständigen Einzelbilder (Dolle-Weinkauff, 2014).

Neben der Ausdifferenzierung zahlreicher Stoffe und Themen für den Comic sind auch neue Spielarten eingeführt worden, welche das Feld des Comics weiter ausbauen. Neben dem ,Manga' auch die seit den 80er-Jahren relative gebräuchliche ,Graphic Novel`. Dolle-Weinkauff zufolge beschreibt letztere jedoch nicht eine eigenständige Gattungsbezeichnung, sondern vielmehr einen literaturkritischen Wertungsbegriff, der literarisch und künstlerisch ,wertvollere' Bildgeschichten als solche hervorheben soll (ebd.). Auch Eders (2016) Auffassung ähnelt dieser. Letztendlich sprächen diese Argumente bei ihr

Kapelari, Suzanne (Hg.), Vierte „Tagung der Fachdidaktik“ 2019:

„Interdisziplinäre fachdidaktische Diskurse zur Bildung für nachhaltige Entwicklung“

(C) 2020 innsbruck university press, ISBN 978-3-99106-019-2, DOI 10.15203/99106-019-2 
allerdings für eine Genrebezeichnung, denn (bloß) für einen Wertungsbegriff. Die Uneinigkeit in der konkreten Klassifizierung findet sich auch in der Einund Abgrenzung dieses Mediums. Es handelt sich bei einer Graphic Novel nicht immer, wie die deutsche Übersetzung vermuten lässt, um einen Roman (oder eine romanhafte Erzählung) (Dolle-Weinkauff, 2012).

Der Begriff ,Graphic Novel' entstand in den 70er-Jahren, der Durchbruch gelang dem Begriff jedoch mit Will Eisner 1978, der sich mit seinem Werk $A$ Contract with God bewusst gegen die ,Profanität‘ des Comics wenden wollte, um aufzuzeigen, dass es sich bei seinem Werk um ein, wahres' Comicbuch handle, welches ,ernsthafte' Literatur an ein adoleszentes oder gar erwachsenes Publikum richte und zwar mit einer erzählerischen Tiefe, die Subtilität und Komplexität bietet (Eder, 2016.).

Merkmale zu finden, die für dieses Format konstituierend sind, gestaltet sich mitunter schwierig. Einige Charakteristika treffen auf Comics genauso zu, andere wiederum finden sich nicht in allen Graphic Novels wieder. Als Beispiel sei hier zunächst die serielle Erscheinungsform angeführt: Selten werden Graphic Novels in serieller Form publiziert (vgl. hierbei das Lustige Taschenbuch mit seiner mittlerweile 500. Ausgabe), doch existieren einige Graphic Novels, die sehr wohl als Serie verfasst und publiziert worden sind (vgl. Spiegelmanns Maus, Sartrapis Persepolis u.e.w.). Vielfach wird den Graphic Novels darüber hinaus ein literarischer Anspruch, den sie mitunter auch explizit erheben, zugeschrieben. Diese Zuweisung erheben allerdings auch eine Vielzahl von Comics (vgl. Das Urteil von Moritz Stetter, das er wiederum ganz bewusst nicht als eine Graphic Novel bezeichnete). Merkmale zu finden, die ausschließlich für Graphic Novels gelten, erschöpfen sich rasch in der hier kurz aufgezeigten verworrenen Beziehung zum Comic. Ähnlich verhält es sich mit der Themenwahl: Die gewählten Motive für Graphic Novels sind ebenso zahlreich wie die ästhetischen Innovationen, mit denen diese dargestellt werden können - auch dieser Umstand kann im gleichen Maße für Comics gelten. Es bleibt daher festzuhalten, dass nach wie vor keine klare Typologie der Graphic Novel existiert und es nur Ansätze einer Klassifikation (vgl. hierzu Baetens \& Frey, 2015) gibt (Eder, 2016.).

Kapelari, Suzanne (Hg.), Vierte „Tagung der Fachdidaktik“ 2019:

„Interdisziplinäre fachdidaktische Diskurse zur Bildung für nachhaltige Entwicklung“

(C) 2020 innsbruck university press, ISBN 978-3-99106-019-2, DOI 10.15203/99106-019-2 


\subsection{Literaturadaptionen in Form von Comics und Graphic Novels}

Schließlich ist es die Etablierung der Graphic Novels, mit der nicht bloß eine allmähliche Anerkennung der Formate insgesamt als Kunstform einhergeht, sondern die auch die Entwicklung der (wie Schmitz-Emans sie nennt) Literaturcomics impulsgebend mitverantwortet (Schmitz-Emans, 2016).

Literaturcomics bezeichnen jene Comics und Graphic Novels, „denen ein literarischer Text zugrunde liegt" (ebd.). Grundsätzlich unterscheiden sich diese Erscheinungsformen durch ihre Nähe zum Ausgangstext sowie durch die Motive für die Wahl der literarischen Vorlage. Dieser Prätext ist zumeist ein weltliterarischer Klassiker (Goethes Faust, Melvilles Moby Dick, Erzähltexte Kafkas u.v.a.), kann aber ebenso einem bestimmten Genre zugeordnet werden, wie die Vorliebe zur Adaption von Schauer- oder Fantasyliteratur (Strokers Dracula u.v.w.) beweist. Als Prätexte fungieren zumeist erzählende und dramatische Werke, während intermediale Übersetzungen von lyrischen Werken eher selten vorzufinden sind (ebd.). Solche Literaturadaptionen in Form von Comics und Graphic Novels sind letztlich das Ergebnis des Transfers eines literarischen Textes, welcher in das ,neue“ Medium übersetzt wird. Dadurch sind diese Transformationen nicht bloß intertextuell, weil sie auf die schriftlichen Bestandteile des Prätextes verweisen, sondern sie sind überdies intermedial, weil ,sie sich aus mindestens zwei verschiedenen medialen Quellen speisen“ (Blank, 2015). Diese Quellen sind verbaler und visueller Natur (hier: Text und Bild) und fallen laut Werner Wolf unter den Punkt der Plurimedialität. Das Wolf'sche System der intermedialen Relationen bietet überdies weitere intermediale Perspektiven auf Literaturadaptionen in Form von Comics und Graphic Novels. So wird die Literaturadaption selbst als intermediale Transposition bezeichnet und umfasst sowohl den Transfer von inhaltlichen als auch formalen Bestandteilen des literarischen Textes (Medium A) in den Comic oder die Graphic Novel (Medium B). Ebenso ist das Medienprodukt häufig durch intermediale Referenzen gekennzeichnet. Diese beschreiben die Übernahme von erzählerischen oder visuellen Mitteln anderer Medienprodukte wie Film oder Werke aus der bildenden Kunst (z.B. Montage, Kameraeinstellungen, Holzschnitt etc.) (Wolf, 2002). Bei Literaturcomics und

Kapelari, Suzanne (Hg.), Vierte „Tagung der Fachdidaktik“ 2019:

„Interdisziplinäre fachdidaktische Diskurse zur Bildung für nachhaltige Entwicklung“

(C) 2020 innsbruck university press, ISBN 978-3-99106-019-2, DOI 10.15203/99106-019-2 
-Graphic Novels treten zumeist alle drei Erscheinungsformen (Plurimedialität, intermediale Transposition, intermediale Referenz) zusammen auf.

\section{Analyse der Literaturadaption}

In der folgenden Analyse beziehe ich mich auf die Literaturadaption Die Verwandlung von Franz Kafka ${ }^{2}$ des französischen Szenaristen Eric Corbeyran sowie des britischen Zeichners Richard Horne. In den Fokus geraten dabei Elemente der Narration und Ästhetik von Comics und Graphic Novels, die insbesondere für Literaturadaptionen gelten, wobei stets auf den Prätext rekurriert wird. Eingeflochten werden Darstellungsmittel, Verfahren und Funktionen dieser Transformation, um das Phänomen der Literaturadaption zu erschließen. Die vorgestellten Kategorien geben einen Einblick in eine größere Analysearbeit und beziehen sich vornehmlich auf jene Module, die Blank 2015 für Literaturadaptionen im Comic vorstellte.

\subsection{Verhältnis zum Prätext}

Die Verwandlung ist nicht bloß eines der Werke Kafkas, mit dem sich Forschende am häufigsten auseinandergesetzt haben, der Großteil der zu Kafkas Oeuvre geführten Kontroversen, beschäftigt sich mit dieser Erzählung. Zahlreiche Deutungsaspekte sind bislang diskutiert worden, der Prätext dieser Graphic Novel bietet für sich betrachtet schon einen reichen Fundus an Interpretationsmöglichkeiten. Auch im schulischen Kontext genießt diese Erzählung kanonischen Status. Corbeyran und Horne haben sich für ihre Adaption demnach eines Textes angenommen, der den Status eines Klassikers innehat und sich damit in der typischen Riege jener intermedialen Übersetzer platziert, die sich mit Transformationen sogenannter ,Weltliteratur beschäftigen. Für dieses Kapitel sind nun jene Deutungsaspekte relevant, bei

2 La Métamorphose im Original. Übersetzung ins Deutsche von Kai Wilksen.

Kapelari, Suzanne (Hg.), Vierte „Tagung der Fachdidaktik“ 2019:

„Interdisziplinäre fachdidaktische Diskurse zur Bildung für nachhaltige Entwicklung“

(C) 2020 innsbruck university press, ISBN 978-3-99106-019-2, DOI 10.15203/99106-019-2 
denen zwischen der Adaption und dem Original die größte Diskrepanz vermutet werden kann.

Das Insekt selbst kann nicht gezeichnet werden. Es kann aber nicht einmal von der Ferne aus gezeigt werden. [...] Wenn ich für eine Illustration selbst Vorschläge machen dürfte, würde ich Szenen wählen, wie: die Eltern und der Prokurist vor der geschlossenen Tür oder noch besser die Eltern und die Schwester im beleuchteten Zimmer, während die Tür zum ganz finsteren Nebenzimmer offen steht (An G.H. Meyer [Kurt Wolff Verlag] 25.10. 1915; B14-17 145).

Kafka wehrte sich stets entschieden gegen eine Illustration des Ungeziefers, wohl um die Unbestimmtheit dieses Wesens bewahrt zu wissen - auch sprach er niemals von einem Käfer oder einer Schabe, sondern von einem Insekt oder Ungeziefer. Dadurch blieben dem Text Unbestimmtheitsgrade erhalten, die zu vielseitigen Interpretationen seitens der Forschung geführt haben (Poppe, 2010) unter anderem auch zu der Frage, ob die Verwandlung in ein Ungeziefer überhaupt stattgefunden hat oder der gesamte Prozess als metaphorisch gelesen werden kann (vgl. hierzu die Ausführungen Fingerhuts, 1994.)

Die Literaturadaption als Graphic Novel hingegen schlägt einen anderen Weg ein, indem sie bereits vor Beginn der Erzählung eine Schabe als Titelbild zeigt (Abb. 1.1) sowie als Einleitung eine Vielzahl von Informationen über das Insekt, die Schabe, die Kakerlake bietet und so das Rätsel um die Gestalt Samsas auflöst, um die absurde Gesamtsituation detailliert erfassen zu können (Blank 2011). 


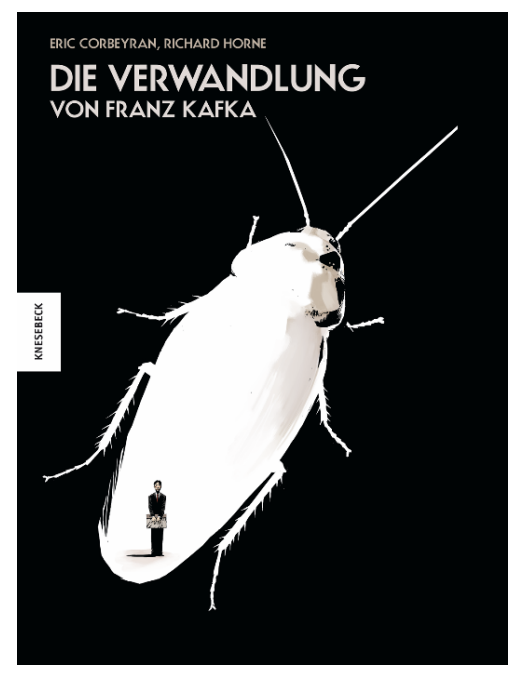

Abb. 1.1 Titelbild der Graphic Novel: Die Verwandlung von Franz Kafka

An dieser Stelle drängt sich nun die Frage auf, wie man diese Adaption noch weiterlesen soll, die sich derartig gegen den Wunsch des Autors wendet. Schließlich bezweckte Kafka mit diesem Verzicht eine gewisse Wirkung, die er beim Lesenden erzeugen wollte. Dass diese auf die Rezipierenden dieser Graphic Novel nun eine vollständig andere ist, wird kaum jemand bezweifeln können. Andererseits macht genau dieser Umstand die Literaturadaption in der Graphic Novel schlussendlich $\mathrm{zu}$ dem, was sie ist und was sie darstellen möchte: etwas Eigenständiges. Der Begriff der Werktreue ist für Adaptionen jedweder Art (sei es die Verfilmung oder der Literatur-Comic) längst überholt (Bohnenkamp, 2005). Eine große Nähe zum Prätext ist nicht automatisch literarisch ,wertvoller' oder, ernster zu nehmen“ als eine Abweichung. Comics und Graphic Novels arbeiten mit eigenen Darstellungsmitteln, die eine explizite Analyse verlangen und die sowohl auf bestehende Deutungen zurückgreifen, als eben auch, so wie beispielsweise durch die explizite Bebilderung des Ungeziefers, neue Aspekte hinzufügen. 
Inwiefern es der Graphic Novel nun weiters gelingt, zentrale Aspekte des Prätextes aufzugreifen und sie in Bild und Text zu übertragen, soll das folgende Beispiel aufzeigen. Trotz der Tragik, welche die Handlung zumeist durchzieht, ist Kafkas Text auf eigentümliche Art und Weise komisch, was unter anderem slapstickartig anmutet (Schmitz-Emans, 2010). So erscheinen die Gedanken Samsas bezüglich seiner bevorstehenden Arbeit und der Probleme aufgrund seiner Verspätung geradezu unsinnig und lustig, bedenkt man die Situation, in der er sich befindet und die im Grunde seine vollständige Aufmerksamkeit verdienen würde. Berechtigt stellt man hier die Frage, wie es der Graphic Novel gelingen kann, solche Elemente der Komik in Bild und Text aufzugreifen. Die Forschung sieht eine solche Möglichkeit der Übertragung durch die Verwendung sogenannter kontrapunktischer Bilder (Zöhrer, 2008). Dabei liefern Text und Bild völlig konträre Informationen zur Narration, wodurch Komik erzeugt werden kann. Die oben zitierte Szene wurde in der Adaption durch kontrapunktische Bild-Text-Kombinationen wiedergegeben. Samsas Gedanken drehen sich um den Chef und den erbarmungslosen Krankenkassenarzt, während die Bilder seinen ekelerregenden Körper zeigen. Die Diskrepanz zwischen dem Gezeigten und dem Beschriebenen soll auch hier Komik erzeugen. Offen bleibt, inwiefern diese ,komische" Intention der Autoren von den Lesenden rezipiert wird.

\subsection{Oberfläche}

Unterschieden wird hier grob zwischen der visuellen und der verbalen Oberfläche der Adaption. Bezüglich der verbalen Gestaltung interessiert vor allem, inwiefern sich die Autoren am Original orientierten oder in Abgrenzung dazu den Prätext modernisierten, abänderten etc. Corbeyran und Horne war offenbar sehr daran gelegen, Kafkas Erzählung textgetreu in das intermediale Format zu übersetzen, wie noch bei vielen anderen Analysekategorien deutlich wird. Ihrer Adaption haftet damit einhergehend etwas Anspruchsvolles an, da sie die Sprachgestalt des Prätextes weitestgehend (abgesehen von Verkürzungen und syntaktischen Angleichungen) bewahrt haben (Blank, 2015). 
Im Hinblick auf die visuelle Oberflächengestaltung sind Fragen der Farbgestaltung und Linienführung relevant (ebd.). Die Bilder selbst sind realistisch gezeichnet, wirken beinahe wie Photographien, wodurch das Geschehen auf den Rezipierenden unmittelbarer wirkt. Typische Oberflächenmerkmale von (klassischen) Comics, wie Onomatopoetika oder verschiedenartige Sprechblasenformen werden so eingesetzt, dass sie Narration und Ästhetik maßgeblich mittragen, was zu einer Darstellung führt, die insgesamt wirklichkeitsnaher wirkt und damit die Ernsthaftigkeit der Erzählung aber auch des gesamten Transformationsprozesses unterstreicht. Das Einfühlen in den Protagonisten wird mit dieser Art der Darstellung, welche die Lesenden nah an das Geschehen ,bindet', trotz seiner Gestalt, die mitunter ekelerregend ist, verstärkt. Die vorherrschende Farbe in der behandelten Literaturadaption ist schwarz, wodurch ein düsteres, zeitgleich aber auch trauriges Bild der Geschehnisse gezeichnet wird. Auch die Schattensetzung ist in dieser Transformation ein bewusstes Mittel der Narration. Die Gesichter der Figuren sind durch tiefe Schatten gekennzeichnet, wodurch die Beschwerlichkeit ihrer Lage hervorgehoben wird. Sie wirken hierdurch traurig, verzweifelt, mitunter auch müde.

Auch Rahmung und Anordnung der Panels können als Träger für Narration und Ästhetik fungieren. Die weiß gezeichneten, geraden Rahmen verleihen der Erzählung eine Unmittelbarkeit und weisen zeitgleich auf die Gegenwärtigkeit der Geschichte hin, da dem Panel-Rahmen die Funktion obliegt, die Erzählzeit zu bestimmen (Schmitz-Emans, 2012). Ein weiterer wichtiger Anhaltspunkt für die Narration ist die Anordnung der Panels. Die Familie selbst sowie der noch nicht verwandelte Gregor sind in geraden, sorgfältig angeordneten Panels zu sehen. Das Ungeziefer hingegen findet sich in schiefen Panels wieder, wodurch der Eindruck verstärkt wird, Samsas Leben sei vollkommen , aus den Fugen geraten'. Auch das störende Moment des Protagonisten wird durch die Panelanordnung deutlich. Die sogenannte Intrusion (Mälzer, 2015) beschreibt das Eindringen von Einzelbildern Samsas in jene Panels, in denen die Familie zu sehen ist - das Ungeziefer stört das normale Familienleben sowohl auf der verbalen als auch auf der visuellen Ebene. Von besonderem Interesse sind

Kapelari, Suzanne (Hg.), Vierte ,Tagung der Fachdidaktik“ 2019:

„Interdisziplinäre fachdidaktische Diskurse zur Bildung für nachhaltige Entwicklung“

(C) 2020 innsbruck university press, ISBN 978-3-99106-019-2, DOI 10.15203/99106-019-2 
überdies die Panels der letzten Seite. Die Familie selbst ist (wie üblich) in geraden Panels gezeichnet, bloß sind diese insgesamt nicht länger lotrecht im Vergleich zur Seite, was die Frage aufwirft, ob hier ein moralisches Urteil gefällt wurde. Die Familie ist zwar offenbar mit sich im Reinen, das tote Ungeziefer schließlich wie Müll entsorgt, doch die Szenerie erscheint bizarr, beinahe sarkastisch, schließlich ist keines der Familienmitglieder über den Verlust des Sohnes sonderlich traurig gestimmt und diesem Umstand wird durch die schiefe Anordnung der in sich geraden Panels Rechnung getragen. Auch das in der Forschung diskutierte sarkastische Moment dieser Szenerie wurde hier aufgegriffen (Poppe, 2010).

Ein für das Modul der Oberfläche ebenso zentrales Darstellungsmittel sind visuelle Leitmotive. Insbesondere die Farbe Rot stellt ein solches Leitmotiv dar und steht in dieser Adaption vor allem für Erotik und Begehren. Die ,Frau im Pelzmantel' als analeptischer panelübergreifender Verweis trägt rot, signalisiert für Samsa als Mensch etwas Begehrenswertes, gleichzeitig aber etwas Unerreichbares; auch als Ungeziefer bleibt die Frau für ihn eine unerfüllbare Wunschvorstellung (schließlich ist er nun als Ungeziefer überhaupt nicht mehr in der Lage, eine Beziehung mit einer ,menschlichen“ Frau einzugehen); die Äpfel, mit denen Samsa grob beworfen wird, sind ebenfalls tiefrot - der Apfel steht hier als Symbol für Versuchung, aber auch Vertreibung. Als Samsa überdies seine Schwester Violine spielen hört, will er sie für sich ganz alleine haben, will sich mit ihr in seinem Zimmer verschließen und sie dabei keineswegs mit den anderen teilen. Diese Begierde wird vor allem dadurch deutlich, dass sich die Farbe des Kleides von beige/braun in den vorangehenden und nachstehenden Panels zu weinrot verwandelt und Samsa an diesem Kleid zu seiner Schwester hochklettert. Auch mit der oben bereits beschriebenen Schlussszene geht ein gewisses Gefühl der Erotik einher. Die sich lasziv in einem purpurroten Kleid streckende Schwester, signalisiert damit ein Stück weit ihre, Geschlechtsreife‘. 


\subsection{Figurendarstellung}

In enger Verbindung zur Handlung steht die Darstellung der Figuren in einer Literaturadaption in Form von Comics und Graphic Novels. Die Festlegung auf eine bestimmte Gestaltung der Akteure ist bereits eine Interpretation, da sie den rein verbalen Text hier visuell konkretisiert (Blank, 2015).

Vergleicht man die Figuren der Graphic Novel von Corbeyran und Horne mit jenen von The Trial (dt. Der Process von Franz Kafka) von Montellier und Mairowitz, so fällt auf, dass sich letztere bei der Figurenzeichnung des Protagonisten stark an Kafka selbst anlehnten, wodurch eine vollkommen andere Interpretation zum Tragen kommt, als in der hier behandelten Literaturadaption von Die Verwandlung (Abb. 1.2-1.3) Nur in wenigen Panels ist Gregor in Menschengestalt zu sehen und hier lässt sich zwar eine gewisse Ähnlichkeit zu Kafka erkennen, doch in weitaus geringerem Ausmaß, sodass diese Adaption nicht automatisch in eine autobiographische Interpretation der Ereignisse überleitet und damit den Deutungshorizont möglicherweise etwas mehr offen lässt, als es Montellier und Mairowitz handhaben, die sich sogar direkt an einem Bild Kafkas (Abb. 1.3) orientierten.

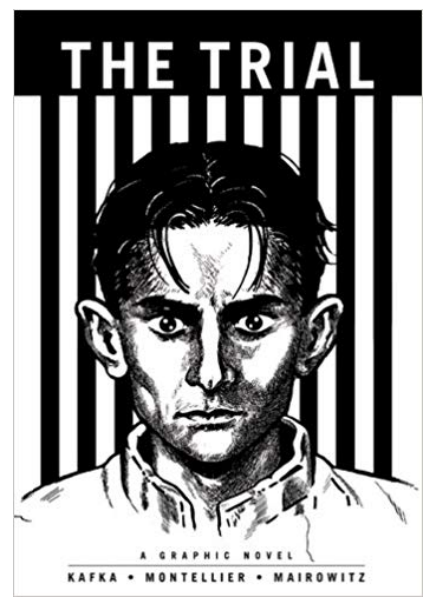

Abb. 1.2 Titelbild des Comics The Trial

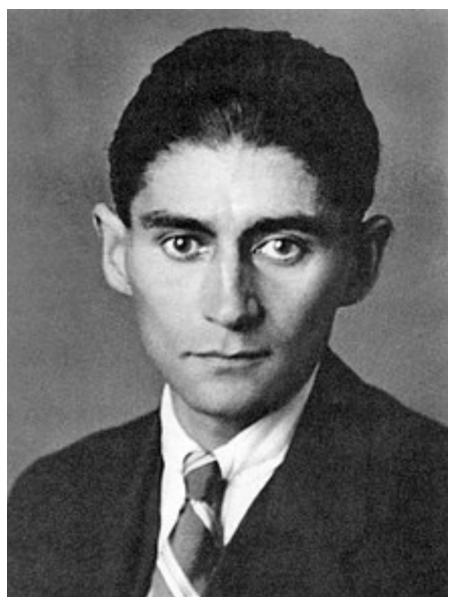

Abb. 1.3 Franz Kafka

Kapelari, Suzanne (Hg.), Vierte „Tagung der Fachdidaktik“ 2019:

„Interdisziplinäre fachdidaktische Diskurse zur Bildung für nachhaltige Entwicklung“

(C) 2020 innsbruck university press, ISBN 978-3-99106-019-2, DOI 10.15203/99106-019-2 
Wie sich Figuren mimisch und gestisch zeigen, kann ebenso Informationen für die Narration liefern. Während der Protagonist, ob seiner Verwandlung in eine Schabe, nur schwer bis gar nicht mimisch oder gestisch wahrnehmbar ist und in diesem Zusammenhang selbst mit seinem Menschenverstand wohl keine sichtbaren menschlichen Attribute mehr aufweist, sind die Gesichter der Familienmitglieder weitaus emotionaler gestaltet. Bis zum Tode Gregors hängen nicht bloß, wie oben bereits beschrieben, tiefe Schatten in den Gesichtern der Familie, sie sind überdies traurig, wütend und schockiert, beinahe jedoch nie fröhlich, solange Samsa noch am Leben ist, was die Tragik der Erzählung weiter verstärkt. Der Trübsinn, den die gesamte Familie ausstrahlt, wird ferner durch die Gestik greifbar. Zumeist sitzen sie gebückt, halten den Kopf gesenkt und wirken ausgelaugt und unverkennbar traurig. Einzig energisch bleibt zuweilen die Schwester, die ihre Wut häufig durch ausladende Gesten mit ihrer Hand zum Ausdruck bringen mag.

Besonders hervorzuheben sind außerdem die Formen der Sprechblasen. Während die Aussagen der übrigen Familienmitglieder in einer runden, abgeflachten Sprechblase platziert wurden, sind Gregors Sprechblasen unruhig und verzerrt. Diese Präzisierung des Prätextes auf visueller Ebene macht deutlich, dass Gregors Stimme sich bis ins Unverständliche verändert hatte:

Gregor erschrak, als er seine antwortende Stimme hörte, die wohl unverkennbar seine frühere war, in die sich aber, wie von unten her, ein nicht zu unterdrückendes, schmerzliches Piepsen mischte, das die Worte förmlich nur im ersten Augenblick in ihrer Deutlichkeit beließ, um sie im Nachklang derart zu zerstören, daß man nicht wußte, ob man recht gehört hatte (Die Verwandlung, 72).

Hierbei nimmt jedoch diese Sprechblasengestaltung der Graphic Novel möglicherweise etwas vorweg, was für den Text eine entscheidende Rolle spielt. Während Gregor nämlich seinen Monolog gegenüber dem Prokuristen hält und ihm versichert, bald wieder auf Reisen zu sein, weiß der/die Lesende des Prätextes noch nicht mit Sicherheit, dass er von Menschen nicht mehr 
verstanden wird, dies löst sich erst am Ende des Monologes auf, als der Prokurist die Flucht ergreift. Durch die Gestaltung der Sprechblasen, die sich doch deutlich von den übrigen Figuren unterscheidet, wird jedoch im Vorfeld schon ersichtlich, dass sich Gregors Stimme verändert hat und nicht mehr menschlich - und damit womöglich nicht länger verständlich - ist.

\subsection{Perspektive}

Wie diese Figuren nun im Raum wahrgenommen werden, ist eine Frage der Perspektive. Obgleich sich dieser Analysepunkt aus mehreren einzelnen Faktoren zusammensetzt, die einer Betrachtung wert wären, ist für diesen Beitrag die Fokalisierung ausschlaggebend. Laut Genette unterscheidet man drei Arten einer möglichen Perspektivierung: die Nullfokalisierung, die das Geschehen ohne perspektivische Einschränkung wiedergibt und somit am ehesten Stanzels auktorialer Erzählsituation entspricht, die interne Fokalisierung (Stanzel spricht hier von der personalen Erzählperspektive), welche nur die Sicht des Protagonisten zeigt, somit zwar Einblick in die Gefühlswelt dieser Figur bieten kann, nicht jedoch alles, was um diese Figur herum geschieht, adäquat erfasst und schlussendlich die externe Fokalisierung, bei der die Sicht auf die Handlung der Erzählung wiedergegeben wird, man jedoch nicht Einblick in die Gefühlswelt der Figuren erhält, was Stanzels neutraler Erzählperspektive am ehesten gleichzusetzen ist (Genette, 1994). Kafkas Die Verwandlung ist weitestgehend (bis auf den Schluss) intern fokalisiert. Die Lesenden sehen das Geschehen aus Gregors Augen und wissen daher nur, was auch Gregor weiß. Das Konzept der internen Fokalisierung lässt sich jedoch nicht ohne weiteres auf den Comic übertragen, schließlich ist es schwierig, einem Comic-Panel tatsächliches Wissen zu unterstellen. Daher fragt man bei Comics und Graphic Novels nach dem ,Wer sieht` nicht auf der verbalen, sondern auf der rein visuellen Ebene (Blank, 2015). Die Literaturadaption von Corbeyran und Horne arbeitet, um es mit Schüwer (2008) auszudrücken, durchaus mit einem halbsubjektiven Bild, d.h. wir sehen dem Protagonisten über die Schulter (in der Filmsprache der, over-the-shoulder-shot ${ }^{`}$ ) und erleben

Kapelari, Suzanne (Hg.), Vierte „Tagung der Fachdidaktik“ 2019:

„Interdisziplinäre fachdidaktische Diskurse zur Bildung für nachhaltige Entwicklung“

(C) 2020 innsbruck university press, ISBN 978-3-99106-019-2, DOI 10.15203/99106-019-2 
dadurch, wie der Protagonist das Geschehen wahrnimmt, sehen (und lesen) die Erzählung aus seinen Augen. Das halbsubjektive Bild ist daher am ehesten mit Genettes interner Fokalisierung gleichzusetzen. Die beiden Abbildungen zeigen die Perspektive Gregors, zumeist die filmtypische Froschperspektive, was einerseits durch die geringe Größe des Ungeziefers erklärbar ist, andererseits auch den Eindruck des Parasiten in der Familie verstärkt, wie er, klein und unnütz geworden, zur Familie aufblicken muss.

Häufig schwenkt das Panel in die extreme Außensicht - bei Genette die Nullfokalisierung - und fokussiert dabei die Familie und ihre Erlebnisse mit dem verwandelten Sohn oder das Insekt selbst in seinem „Menschenzimmer“ (zumeist aus verzerrter Perspektive). Damit wird ersichtlich, wie ,verrückt ${ }^{6}$ das Geschehen ist. Ohne erkennbaren Beobachter wird das verwandelte Insekt gezeigt, sein Zimmer ist schief, seine Fühler überlang, die Decke des Raumes nicht ersichtlich, das Groteske der Verwandlung wird durch die Perspektive besonders betont. Obgleich der Prätext aufgrund seiner rein internen Fokalisierung diese Sicht auf das Geschehen nicht einnehmen kann, so bietet er doch Potenzial für diese Art der Darstellung. Der (berühmte) erste Satz der Erzählung lautet nämlich: „Als Gregor Samsa eines Morgens aus unruhigen Träumen erwachte, fand er sich in seinem Bett zu einem ungeheuren Ungeziefer verwandelt" (Die Verwandlung, 69). Durch die durchwegs eigenartige Formulierung „fand er sich“ ist auch diese Art der bildlichen Darstellung nicht ganz von der Hand zu weisen. Wie eine außerkörperliche Erfahrung „fand“ Gregor sich verwandelt vor.

Ein anderer Blick von außen befindet sich außerhalb der diegetischen Welt der Adaption auf der Innenseite des Einbandes. Den Rezipierenden wird schon vor der Lektüre die Gestalt Gregors mitgeteilt, eine Information, die aus Gregors Sicht nicht möglich ist und die auch bei Kafka selbst stets ein Rätsel geblieben ist. D.h. die Lesenden wissen bereits zu Beginn der Erzählung, mehr ‘̈ über die Verwandlung als der Protagonist, was bei der rein internen Fokalisierung und dem expliziten Verzicht auf Illustrationen im Prätext nicht möglich ist. Des Weiteren ist auch dann eine Nullfokalisierung gegeben, wenn Ausschnitte aus

Kapelari, Suzanne (Hg.), Vierte „Tagung der Fachdidaktik“ 2019:

„Interdisziplinäre fachdidaktische Diskurse zur Bildung für nachhaltige Entwicklung“

(C) 2020 innsbruck university press, ISBN 978-3-99106-019-2, DOI 10.15203/99106-019-2 
Prag gezeigt werden. Diese dienen einerseits dazu, das Geschehen zu kontextualisieren (Blank, 2015), also in Prag zu verorten, andererseits auch dazu, die von Kafka vorgegebene Einteilung in drei Kapitel deutlich zu machen. Das zweite Kapitel beginnt mit der Sicht auf die Dächer Prags, in einer Nacht, der Mond scheint nur hie und da durch die dichte Wolkendecke hindurch. Mit Beginn des dritten Kapitels ist ein anderer Stadtteil Prags zu sehen und die Dämmerung hat bereits eingesetzt, der Himmel ist rötlich-orange, im rechten Eck kann sogar das Licht der Sonne entdeckt werden. Die letzten Seiten sind schlussendlich bei Tageslicht dargestellt, vor allem als Gregor stirbt, ist die Nacht ,endgültig' vorüber und mit ihr das Problem der übrigen Familie Samsa, die glücklich in der Straßenbahn sitzt. Die durchbrochene Nacht bringt auch (endlich) wieder Sonne und damit Freude in das Leben der übrigen Familie Samsa.

Ist der Blick auf die übrigen Familienmitglieder gerichtet und Gregor kann als Betrachter ausgeschlossen werden, spricht man laut Genette von externer Fokalisierung. Dies dient unter anderem dazu, das Verhalten und die Gefühle der übrigen Figuren $\mathrm{zu}$ registrieren. Die Bilder zeigen, dass hier nicht ,nullfokalisiert` wird, sondern vielmehr aus Sicht eines Beobachters gezeigt wird, der sich nicht sichtbar im Raum mit den übrigen Figuren aufhält und so den Rezipierenden einen Blick auf die Familie bietet, der sich dem Protagonisten verschließt. 


\section{Zur geplanten empirischen Studie und deren didaktischem Horizont}

Da der Fokus dieses Beitrags vermehrt auf literaturwissenschaftlicher Analyse lag, könnte die Frage aufkommen, worauf ich damit in didaktischer Hinsicht verweisen möchte. Da in empirischer Hinsicht dem Comic und der Graphic Novel noch verhältnismäßig wenig Beachtung geschenkt wurde, möchte ich mit meiner qualitativen Studie diesem Missstand entgegentreten. Ob und inwiefern sich nämlich die Rezeption von Literaturadaptionen und deren Prätexten unterscheidet, und wie Phänomene der Narration in den jeweiligen Medien wahrgenommen werden, wird diese Studie offen legen. Mithilfe der Methode des Lauten Denkens rezipiert eine Gruppe von SchülerInnen der Sekundarstufe II einen Ausschnitt aus dem Erzähltext Kafkas, die andere Gruppe einen Ausschnitt aus der Adaption. Die daraus abzuleitenden Erkenntnisse sollen Aufschluss darüber geben, wie die beiden rezipiert werden und wie ein entsprechend informierter Unterricht auf Hilfen beim jeweiligen Textverständnis reagieren kann. Wie kann das eine Medium das Verständnis des anderen erhellen und vice versa? Wie kann durch den Einsatz des jeweiligen Mediums die Lesemotivation gesteigert werden? Dabei steht nicht bloß der Vergleich im Mittelpunkt der Analyse, sondern die relativ offen formulierte Frage, wie gewisse literarische Phänomene, die auch dieser Beitrag ausgelotet hat, in den jeweiligen Medien wahrgenommen werden. Das Ziel der Studie ist es nicht, eine ,bessere' Herangehensweise zu Literatur zu finden, sondern eine gegenseitige Ergänzung und Befruchtung $\mathrm{zu}$ finden - eine , $\mathrm{Axt}^{6}$, die durch verschiedene Verwendungsmöglichkeiten einen nachhaltigen Literaturunterricht erzielt. 


\section{Resümee}

Nachhaltiges literarisches Lernen kommt nicht an der sich stetig ändernden außerschulischen literarischen Wirklichkeit vorbei. Comics und Graphic Novels sind bereits in vielerlei Hinsicht Teil der literarischen Kultur. Der Buchhandel und die Bibliothek bieten eigene Abteilungen an, viele Buchmessen integrieren diese Art der Literatur bereitwillig in ihr Programm. Der Literaturunterricht tut sich bislang jedoch schwer damit, einen sinnvollen Zugang zu diesen Medienformaten zu finden, was auch an den Lehrbüchern liegt, die Comics und Graphic Novels nur marginal bis gar nicht aufgreifen. Nichtsdestotrotz sollte hier freilich nicht ein blindes Aufnehmen sämtlicher Adaptionen in den Vordergrund gerückt werden, sondern vielmehr ist es mir ein Anliegen, die vielseitigen Möglichkeiten aufzuzeigen, wie man in den Genuss von Literatur kommen kann und wie fruchtbar sich eine Analyse und damit einhergehend eine Deutung gestalten kann.

Mit eigenen Darstellungsmitteln und zahlreichen Möglichkeiten, Narration und Ästhetik mithilfe von Text und Bild zu gestalten, gelingt es vielen Comics und Graphic Novels, Prätexte geschickt zu adaptieren. Dies führt mitunter auch zur unangebrachten Hoffnung, solche Adaptionen würden die Lesenden , an die Originalwerke" heranführen, wofür jedoch keine empirischen Beweise vorliegen. Darüber hinaus gilt, unterhaltende" Literatur dann als ,fertig gelesen“, wenn die Handlung weitestgehend nachvollzogen wurde. Die Ansicht, eine Literaturadaption könnte als „Einstiegsmedium“ (Blank, 2015) fungieren, kann in Bezug auf diese Transformation nicht gelten, schließlich kann die Handlung von Die Verwandlung durch die Adaption gut verstanden werden. Es bleibt der Appell, Literaturadaptionen in Form von Comics und Graphic Novels als eigenständige Kunstwerke zu betrachten, die weder dem Prätext genauestens entsprechen, noch als sein Sprungbett fungieren müssen.

Bildung für nachhaltige Entwicklung bedeutet für den Literaturunterricht einerseits das Aufgreifen aktueller Themen wie Toleranz, Offenheit, Akzeptanz u.v.w. Andererseits kann Literaturunterricht auch auf einer tiefergreifenden Ebene für Nachhaltigkeit sorgen, indem die außerschulische literarische Realität in den Unterricht integriert wird, um so ein nachhaltiges literarisches Lernen zu

Kapelari, Suzanne (Hg.), Vierte ,Tagung der Fachdidaktik“ 2019:

„Interdisziplinäre fachdidaktische Diskurse zur Bildung für nachhaltige Entwicklung“

(C) 2020 innsbruck university press, ISBN 978-3-99106-019-2, DOI 10.15203/99106-019-2 
ermöglichen. Diese Form der Nachhaltigkeit bezieht sich dabei eben nicht auf explizite Themen der BNE, sondern auf das Herstellen einer inneren, bewussten Form der Nachhaltigkeit, die sich durch intensive, subjektorientierte Lektüre definiert und damit schlussendlich Nachhaltigkeit herstellt.

\section{Literatur}

Primärliteratur

Kafka, F. (2017). Die Verwandlung. In M. Müller (Eds.). Franz Kafka. Erzählungen (pp. 67-132). Stuttgart: Reclam. (=Universalbibliothek, 9426).

Corbeyran, E., Horne, R. (2010). Die Verwandlung. Von Franz Kafka. Übersetz. von Kai Wilksen. München: Knesebeck.

Sekundärliteratur

Baetens J., Frey, H. (2015). The Graphic Novel. An Introduction. Cambridge: Cambridge University Press.

Blank, J. (2015). Literaturadaptionen im Comic. Ein modulares Analysemodell. In M. Schmitz-Emans \& D. Grünewald (Eds.), Bildnarrative, Studien zu Comics in Bilderzählungen. Berlin: Bachmann.

Blank, J. (2011). Erzählperspektive im Medienwechsel. Visuelle Fokalisierung in Comic-Adaptionen von Texten Franz Kafkas. https://edoc.huberlin.de/bitstream/handle/18452/8106/blank.pdf?sequence=1\&isAllowed=y

Bohnenkamp, A. (2005). Literaturverfilmungen als intermediale Herausforderung. Ein hybrides Genre. In A. Bohnenkamp (Eds.), Literaturverfilmungen (pp. 9-40). Stuttgart: Reclam. (=Universal-Bibliothek, 17536).

Dolle-Weinkauff, B. (2014). Comic, Manga, Graphic Novel. In A. Tillmann et al. (Eds.), Handbuch Kinder und Medien (pp. 457-468). Wiesbaden: Springer Fachmedien. (=Digitale Kultur und Kommunikation, 1).

Eder, B. (2016). Graphic Novels. In J. Abel \& C. Klein (Eds.), Comics und Graphic Novels. Eine Einführung (pp. 156-168). Stuttgart: Metzler.

Fingerhut, K. (1994). Die Verwandlung. In M. Müller (Eds.), Interpretationen. Franz Kafka: Romane und Erzählungen (pp. 42-74). Stuttgart: Reclam. (=UniversalBibliothek 17521).

Genette, G. (1994). Die Erzählung. Übersetz. v. Andreas Knop. München: UTB. (=UniTaschenbücher, 8083). 
Kafka, F. (1966). Briefe 1902-1924. In M. Brod (Eds.). Frankfurt a.M.: Fischer.

Mälzer, N. (2015). Taxonomien von Bild-Text-Beziehungen im Comic. In N. Mälzer (Eds.), Comics - Übersetzungen und Adaptionen (pp. 47-64). Berlin: Frank \& Timme.

Montellier, C.; Mairowitz, R. Z. (2008). The Trial. A Graphic Novel. New York, London: Sparknotes.

Palmier, J.P. (2015). Strukturverwandte. Zur Adaptierbarkeit von Erzählungen in Literatur, Comic und Film. In N. Mälzer (Eds.), Comics - Übersetzungen und Adaptionen (pp. 81-96). Berlin: Frank \& Timme.

Poppe, S. (2010). Die Verwandlung. In M. Engel \& B. Auerochs (Eds.), KafkaHandbuch. Leben - Werk - Wirkung (pp. 164-174). Stuttgart, Weimar: J.B. Metzler.

Schmitz-Emans, M. (2016). Literaturcomics. In J. Abel \& C. Klein: Comics und Graphic Novels. Eine Einführung (pp. 276-290). Stuttgart: Metzler.

Schmitz-Emans, M. (2012). Literatur-Comics. Adaptationen und Transformationen der Weltiteratur. Berlin. Walter de Gruyter.

Schmitz-Emans, M. (2010). Franz Kafka. Epoche - Werk-Wirkung. In W. Barner \& G. E. Grimm (Eds.). München: C.H.Beck.

Schüwer, M. (2008). Wie Comics erzählen. Grundriss einer intermedialen Erzähltheorie der grafischen Literatur. Trier: WVT.

Stanzel, F. K. (1993). Typische Formen des Romans. Göttingen: Vandenhoeck u. Ruprecht.

Wolf, W. (2002). Intermediality Revisited. Reflections on Word and Music Relations in the Context of a General Typology of Intermediality. In S. M. Lodato et al. (Eds.), Word and Music Studies. Essays in Honor of Steven Paul Scher and on Cultural Identity and the Musical Stage (pp. 13-34). Amsterdam: Rodopi.

Zöhrer, M. (2008). Weltliteratur im Bilderbuch. In E. Seibert und H. Lexe (Eds). Wien: praesens.

Abbildungsverzeichnis

Abb. 1.1 Corbeyran, Eric; Horne, Richard (2010). Die Verwandlung. Von Franz Kafka. Übersetz. von Kai Wilksen. München: Knesebeck.

Abb. 1.2 Montellier, Chantal; MAIRowitz, Robert Z. (2008): The Trial. A Graphic Novel. New York, London: Sparknotes.

Abb. 1.3; Porträt Franz Kafkas aus dem Jahre 1923. URL:

https://de.wikipedia.org/wiki/Franz Kafka [zuletzt aufgerufen am 10. April 2019].

Kapelari, Suzanne (Hg.), Vierte ,Tagung der Fachdidaktik“ 2019:

„Interdisziplinäre fachdidaktische Diskurse zur Bildung für nachhaltige Entwicklung“

(C) 2020 innsbruck university press, ISBN 978-3-99106-019-2, DOI 10.15203/99106-019-2 
